\title{
Nivolumab versus chemotherapy in Japanese patients with advanced esophageal squamous cell carcinoma: a subgroup analysis of a multicenter, randomized, open-label, phase 3 trial (ATTRACTION-3)
}

\author{
Masanobu Takahashi ${ }^{1} \cdot$ Ken Kato $^{2} \cdot$ Morihito Okada $^{3} \cdot$ Keisho Chin $^{4} \cdot$ Shigenori Kadowaki $^{5} \cdot$ Yasuo Hamamoto $^{6}$. \\ Yuichiro Doki $^{7} \cdot$ Yutaro Kubota $^{8} \cdot$ Hisato Kawakami $^{9} \cdot$ Takashi Ogata $^{10} \cdot$ Hiroki Hara $^{11} \cdot$ Manabu Muto $^{12}$. \\ Yuichiro Nakashima ${ }^{13} \cdot$ Ryu Ishihara $^{14} \cdot$ Masahiro Tsuda $^{15} \cdot$ Satoru Motoyama ${ }^{16} \cdot$ Mamoru Kodani $^{17} \cdot$ Yuko Kitagawa $^{18}$
}

Received: 31 August 2020 / Accepted: 23 October 2020 / Published online: 10 November 2020

(c) The Author(s) 2020

\begin{abstract}
Background The efficacy and safety of nivolumab versus chemotherapy was evaluated in the Japanese subpopulation from the overall intent-to-treat (ITT) population of the ATTRACTION-3 trial conducted in patients with advanced esophageal squamous cell carcinoma (ESCC) as second-line treatment.

Methods Data from Japanese patients enrolled in the multicenter, randomized, open-label, phase 3 ATTRACTION-3 trial were analyzed. The primary endpoint was overall survival (OS). Secondary endpoints included duration of response (DOR), objective response rate (ORR), disease control rate (DCR), and safety. Exploratory subgroup analyses evaluated the association between OS and stratification factors/baseline variables.

Results Overall, 274 (nivolumab, 136; chemotherapy, 138) of the 419 patients in ATTRACTION-3 were enrolled from Japan: response-evaluable population $(107 ; 108)$ and safety population $(135 ; 138)$. OS tended to be longer in the nivolumab group versus the chemotherapy group (median: 13.4 months vs. 9.4 months; HR, 0.77; 95\% CI 0.59-1.01). Median DOR was longer in the nivolumab group (7.6 months) versus the chemotherapy group (3.6 months). ORRs were similar between the nivolumab [22.4\% of patients (24/107)] and chemotherapy groups [22.2\% (24/108); odds ratio, 0.98; 95\% CI 0.52-1.87]. DCR was lower in the nivolumab group [41.1\% (44/107)] versus the chemotherapy group [66.7\% (72/108)]. OS in the exploratory analysis consistently favored the nivolumab group versus the chemotherapy group. Overall, nivolumab demonstrated favorable efficacy and safety versus chemotherapy in the Japanese subpopulation, and the trend was similar to that observed in the overall ATTRACTION-3 ITT population.

Conclusion Nivolumab represents a new standard second-line treatment option for Japanese patients with advanced ESCC.
\end{abstract}

Keywords Esophageal squamous cell carcinoma $\cdot$ Japanese population $\cdot$ Nivolumab $\cdot$ ATTRACTION-3

\section{Introduction}

Metastatic esophageal cancer has a poor prognosis, with a 5 -year relative survival rate of $<8 \%$ globally, including in Japan $[1,2]$. Esophageal squamous cell carcinoma (ESCC)

Electronic supplementary material The online version of this article (https://doi.org/10.1007/s10388-020-00794-x) contains supplementary material, which is available to authorized users.

Masanobu Takahashi

masanobu.takahashi.a7@tohoku.ac.jp

Extended author information available on the last page of the article is the dominant histological subtype worldwide ( 90\%) [3, 4]. Furthermore, differences in baseline characteristics are observed between Japanese and Western patients with esophageal cancer; the incidence of squamous cell carcinoma is higher in Japanese patients than in Western patients [5]. In Japan, fluoropyrimidine plus platinum compounds are used as first-line therapy and taxanes as second-line therapy in patients with unresectable esophageal cancer $[6,7]$.

Globally, current second-line chemotherapy options for ESCC offer poor long-term survival [7-12]. Therefore, new therapeutic approaches are warranted for patients with advanced ESCC. Immune checkpoint inhibition is one such 
strategy successfully evaluated in many cancers. The efficacy and safety of nivolumab (an immune checkpoint inhibitor [ICI]) has been demonstrated in esophageal cancer, with a favorable 2-year overall survival (OS) in Japanese patients $(17.2 \%)$ in the phase 2, single-arm ATTRACTION-1 trial $[12,13]$ and with significant prolongation of OS [median OS, 10.9 months vs. 8.4 months; hazard ratio (HR) for death, $0.77 ; p=0.019$ ] in the global, phase 3 , randomized, ATTRACTION-3 trial compared with chemotherapy [14]. A smaller proportion of patients in the nivolumab group [38/209 (18\%)] experienced grade 3/4 treatment-related adverse events (TRAEs) versus those in the chemotherapy group [131/208 (63\%)] in ATTRACTION-3 [14].

Based on ATTRACTION-1 and ATTRACTION-3 results, nivolumab was approved in Japan for the treatment of patients with unresectable advanced or recurrent esophageal cancer on February 21, 2020 [15]. The Japanese esophageal cancer practice guidelines state that second-line chemotherapy for Japanese patients with esophageal cancer has been evaluated in a small sample size in phase 2 studies only, with no evidence of clear efficacy from any reports [6, 7]. Therefore, it is meaningful to evaluate the efficacy and safety of nivolumab in the Japanese population. Hence, this subgroup analysis was performed to evaluate the efficacy and safety of nivolumab versus chemotherapy in the Japanese subpopulation, and to assess whether the results in the Japanese population are similar to those in the overall intentto-treat (ITT) population of ATTRACTION-3, which was conducted in patients with advanced ESCC [14].

\section{Methods}

\section{Study design and patients}

Data from Japanese patients enrolled in the multicenter, randomized, open-label, phase 3 ATTRACTION-3 trial were analyzed. The results were compared and discussed with the outcomes from the overall study population (enrolled at 90 hospitals across Denmark, Germany, Italy, Japan, South Korea, Taiwan, the UK, and the USA), with November 12, 2018, as the data cutoff date for comparison. The study design and overall results have been previously reported [14]. Briefly, patients aged $\geq 20$ years with unresectable esophageal cancer whose major current or previously resected lesion was in the cervical or thoracic esophagus (including the esophagogastric junction) and was pathologically confirmed as squamous or adenosquamous cell carcinoma were enrolled. Eligible patients were refractory or intolerant to fluoropyrimidine- and platinum-based chemotherapy, had previously received one treatment regimen, were not indicated for a radical resection, and had a life expectancy of $\geq 3$ months.
Totally, 419 patients were enrolled in the overall study population and randomly assigned (1:1) to receive nivolumab $(n=210)$ or the investigator's choice of chemotherapy [paclitaxel (PTX) or docetaxel (DTX), $n=209$ ]. Randomization was performed using an interactive web response system (block size $=4$ ), and patients were stratified according to their geographical location (Japan vs the rest of the world), number of organs with metastases $(\leq 1$ vs. $\geq 2$ ), and expression of programmed death-ligand 1 (PDL1: $<1 \%$ vs. $\geq 1 \%$ ).

The study was performed in accordance with the Good Clinical Practice guidelines developed by the International Council for Harmonisation and approved by the institutional review board or independent ethics committee at each study site. All patients provided written informed consent before enrollment.

\section{Treatment}

Nivolumab was administered at $240 \mathrm{mg}$ intravenously (IV) over 30 min every. 2 weeks (each cycle was 6 weeks long). PTX was administered at $100 \mathrm{mg} / \mathrm{m}^{2}$ IV for $\geq 60 \mathrm{~min}$ once per week for 6 weeks followed by 1 treatment-free week (each cycle was 7 weeks long), and DTX was administered at $75 \mathrm{mg} / \mathrm{m}^{2}$ IV for $\geq 60$ min every 3 weeks (each cycle was 3 weeks long), until disease progression or unacceptable toxicity.

Treatment that was interrupted or delayed due to adverse events (AEs) was resumed when patients met the protocoldefined criteria for treatment resumption. Per protocol, pre-specified dose reductions were permitted for toxicities related to PTX and DTX. Dose reductions were prohibited in the nivolumab group. Additional details pertaining to study procedure have been reported previously [14].

\section{Assessments}

Tumor assessments were performed using computed tomography or magnetic resonance imaging per Response evaluation criteria in solid tumors (RECIST) version 1.1 at baseline, after each 6-week cycle for 1 year, and every 12 weeks thereafter until initiation of post-study treatment, disease progression, or recurrence. Complete response (CR) and partial response (PR) were confirmed by $\geq 2$ successive scans within a minimum of 4 weeks. Tumor cell PD-L1 expression was assessed by a central laboratory using immunohistochemistry (PD-L1 IHC 28-8 pharmDx assay; Dako, an Agilent Technologies company, Santa Clara, CA, USA).

Exploratory subgroup analyses evaluated the association between OS and stratification factors or baseline variables. The pre-specified exploratory endpoint assessed the healthrelated quality of life (HRQoL) based on the three-level version of the EuroQol 5D questionnaire (EQ-5D-3L). 
AEs were assessed according to the National Cancer Institute Common Terminology Criteria for Adverse Events (CTCAE) version 4.0 throughout the treatment period and for 28 days after the end of treatment. Serious AEs were assessed throughout the study period and for 100 days after treatment discontinuation. Other AEs data were collected for 28 days after treatment discontinuation.

\section{Outcomes}

The primary endpoint was OS. Secondary endpoints included the proportion of patients with an investigatorassessed objective response rate [ORR; the percentage of patients whose best overall response (BOR) was either a CR or a PR], BOR, progression-free survival (PFS), proportion of patients with disease control $[\mathrm{CR}+\mathrm{PR}+$ stable disease (SD)], maximum percentage change from baseline in the sum of target lesion diameters, time to response (time from randomization to the first confirmed CR/PR), duration of response (DOR; time from the first response to the first documented tumor progression or death), and safety. Pre-specified exploratory subgroup analyses assessed the association between OS and stratification factors or baseline variables, including PD-L1 expression $(<1 \%, \geq 1 \%,<5 \%, \geq 5 \%,<10 \%$, and $\geq 10 \%)$, age ( $<65$ years vs. $\geq 65$ years), sex, race (Asian vs white), Eastern Cooperative Oncology Group (ECOG) performance status (PS; 0 vs. 1), prior surgery, prior radiotherapy, and history of smoking. As a pre-specified exploratory endpoint, HRQoL was assessed based on EQ-5D-3L, comprising the visual analog scale (VAS) and descriptive system, to generate the utility index. Assessments were performed every 6 weeks from the start of cycle 1 until the end of the treatment phase and every 12 weeks thereafter. Further details have been reported previously [14].

\section{Statistical analysis}

OS and PFS analyses were performed in the Japanese subpopulation, defined as all patients who were randomly assigned to the study treatment. ORR, BOR, disease control rate (DCR), time to response, and DOR were assessed in all randomized patients in the Japanese subpopulation who had target lesion measurements at baseline (i.e., the responseevaluable population). Safety was assessed in all patients in the Japanese subpopulation who received $\geq 1$ dose of the assigned treatment (safety population). Both descriptive and mixed-effect model for repeated measure (MMRM) analyses of patient-reported outcomes were performed for all randomized patients in the Japanese subpopulation who had an EQ-5D-3L and utility index assessment at baseline and $\geq 1$ post-baseline assessment including unscheduled or follow-up visits (i.e., patient-reported outcomes population).
Estimates of median OS, PFS, and DOR were derived from the Kaplan-Meier (KM) estimates, and the corresponding two-sided $95 \%$ confidence intervals (CIs) were calculated using the Brookmeyer and Crowley method based on a $\log -\log$ transformation. The stratified Cox proportional hazards regression model, with randomization factors as stratification factors and treatment group as a single covariate, was used to assess differences between the treatment groups for OS and PFS; a two-sided stratified log-rank test using randomization stratification factors with a $5 \%$ significance level was used. Further details are presented as footnotes or have been published previously [14]. Statistical analyses were performed using the SAS software (version 9.4; SAS Institute, Cary, NC, USA).

\section{Results}

Overall, 274 (nivolumab, 136; chemotherapy, 138) of the 419 patients in ATTRACTION-3 were enrolled from 45 study sites in Japan. The response-evaluable population comprised 215 patients (nivolumab, 107; chemotherapy, 108); the safety population comprised 273 patients (nivolumab, 135; chemotherapy, 138). At database lock (November 12, 2018), the minimum follow-up period was 17.6 months. Baseline characteristics of the patients were well balanced between the treatment groups (Table 1). Overall, $83.0 \%$ and $27.5 \%$ of patients received $90 \%$ to $<110 \%$ of nivolumab and

Table 1 Patient demographics and baseline characteristics

\begin{tabular}{lll}
\hline Trial & \multicolumn{2}{l}{ Japanese subpopulation } \\
\cline { 2 - 3 } Characteristic, $n(\%)$ & Nivolumab $n=136$ & Chemotherapy $n=138$ \\
\hline $\begin{array}{l}\text { Age, median (range), } \\
\quad \text { years }\end{array}$ & $65.0(41-82)$ & $68.0(33-80)$ \\
Age $\geq 65$ years & $76(55.9)$ & $98(71.0)$ \\
Sex, male & $113(83.1)$ & $117(84.8)$ \\
ECOG PS 0 & $83(61.0)$ & $88(63.8)$ \\
ECOG PS 1 & $53(39.0)$ & $50(36.2)$ \\
Recurrent EC & $65(47.8)$ & $70(50.7)$ \\
Number of organs with metastases (IWRS source) \\
$\leq 1$ & $65(47.8)$ & $66(47.8)$ \\
$\geq 2$ & $71(52.2)$ & $72(52.2)$ \\
Prior surgery & $68(50.0)$ & $69(50.0)$ \\
Prior radiotherapy & $99(72.8)$ & $89(64.5)$ \\
PD-L1 expression & & $35(25.4)$ \\
$\geq 10 \%$ & $41(30.1)$ & $46(33.3)$ \\
$\geq 5 \%$ & $46(33.8)$ & $68(49.3)$ \\
$\geq 1 \%$ & $66(48.5)$ &
\end{tabular}

$E C$ esophageal cancer; ECOG PS Eastern Cooperative Oncology Group performance status; IWRS interactive web response system; $P D-L 1$ programmed death-ligand 1 
chemotherapy planned relative dose intensity, respectively, in the Japanese subpopulation (Supplementary Table 1).

At database lock, study treatment was permanently discontinued in 124/135 (91.9\%) patients in the nivolumab group and 135/138 (97.8\%) patients in the chemotherapy group in the Japanese subpopulation; reasons for treatment discontinuation (nivolumab vs chemotherapy) were disease progression [86 (63.7\%) vs. $98(71.0 \%)$, worsening of clinical symptoms judged as progressive disease [PD; 15 (11.1\%) vs. 8 (5.8\%)], onset of grade $\geq 2$ interstitial lung disease [9 $(6.7 \%)$ vs. $4(2.9 \%)]$, treatment withheld for $>6$ weeks due to AEs [3 (2.2\%) vs. $2(1.4 \%)$ ], onset of grade $\geq 3$ peripheral neuropathy $[0(0 \%)$ vs. $2(1.4 \%)]$, drug-related liver function test abnormality [1 $(0.7 \%)$ vs $0(0 \%)]$, onset of grade $\geq 3$ hypersensitivity (eg, diarrhea, colitis, neurologic toxicity, hypersensitivity reaction, infusion reaction) $[1(0.7 \%)$ vs. $0(0 \%)$, three rounds of dose reductions $[0(0 \%)$ vs. 4 $(2.9 \%)$ ], physician's discretion [9 (6.7\%) vs. $13(9.4 \%)$ ], and other reasons [5 (3.7\%) vs. 8 (5.8\%)]. After study treatment discontinuation, the proportion of patients in the Japanese subpopulation who received subsequent anticancer treatment was higher in the nivolumab group [58.8\% (80/136)] than in the chemotherapy group [47.1\% (65/138)]. The proportion of patients receiving taxanes as subsequent anticancer therapy in the nivolumab group was high in the Japanese subpopulation [56.6\% (77/136); Supplementary Table 2]. Further details of subsequent anticancer treatment in the Japanese subpopulation are presented in Supplementary Table 2.

\section{Efficacy in the Japanese subpopulation}

\section{Overall survival}

Median follow-up was 13.21 months [interquartile range (IQR), 6.11-19.48; $n=136$ ] in the nivolumab group and 8.74 months (IQR, 5.06-17.84; $n=138$ ) in the chemotherapy group. By KM analysis, OS was numerically longer in the nivolumab group versus the chemotherapy group [median: 13.4 months vs. 9.4 months; HR, 0.77 (95\% CI 0.59-1.01); Fig. 1a]. In the subgroup analysis, OS was numerically longer in the nivolumab group consistently versus the chemotherapy group in the Japanese subpopulation (Fig. 2 and Supplementary Fig. 1). In the Japanese ITT subpopulation, as of November 2018, five deaths had occurred due to TRAEs (nivolumab, 2/135; chemotherapy, 3/138).

\section{Progression-free survival}

By KM analysis, the median PFS was 2.7 months (95\% CI 1.61-2.99) in the nivolumab group versus 3.8 months ( $95 \%$ CI 2.99-4.21) in the chemotherapy group (HR, 1.03; 95\% CI $0.80-1.33$; Fig. 1b).

\section{Response}

Patients with confirmed CR or PR to nivolumab showed a median time to response of 2.74 months (minimum-maximum, 1.3-6.5). The median DOR was numerically longer in the nivolumab group (7.6 months; 95\% CI 4.4-11.1) versus the chemotherapy group (3.6 months; $95 \%$ CI 2.8-4.2; Fig. 1c). ORRs were similar between the nivolumab [22.4\% (24/107)] and chemotherapy groups [22.2\% (24/108); odds ratio, 0.98 ; 95\% CI $0.52-1.87]$. DCR was lower in the nivolumab group [41.1\% (44/107)] versus the chemotherapy group [66.7\% (72/108); odds ratio, 0.30; 95\% CI 0.17-0.55; Table 2 a].

\section{Tumor burden}

The best change from baseline in target lesion size in the nivolumab group versus the chemotherapy group is presented in Supplementary Fig. 2.

\section{PD-L1 expression status}

Baseline tumor samples for determination of PD-L1 were available for all patients in the Japanese subpopulation. The median OS in patients with $<1 \%$ versus $\geq 1 \%$ tumor PD-L1 expression was 13.4 months (95\% CI 8.84-17.05) versus 12.75 months (9.92-17.84), respectively, with nivolumab and 10.32 months (7.39-13.67) versus 8.38 months (5.98-9.89), respectively, with chemotherapy. The prespecified interaction analysis indicated no significant interaction of treatment effect by PD-L1 status in the Japanese subpopulation (Supplementary Fig. 3). The median OS in the Japanese subpopulation based on tumor PD-L1 expression $(<1 \%$ vs. $\geq 1 \%)$ was similar to that in the overall ITT population.

\section{Quality of life}

The proportion of patients completing the EQ-5D-3L questionnaires exceeded $87 \%$ in both groups through week 42 . In the Japanese subpopulation, the on-treatment improvement in quality of life (QoL) of patients was more favorable in the nivolumab group than in the chemotherapy group (Supplementary Fig. 4). This improvement in QoL was similar to that observed in the overall ITT population [14].

\section{Safety}

TRAEs of any grade and grade 3-5 were observed in a lower proportion of patients in the nivolumab group $(68.1 \%$, $17.0 \%)$ versus the chemotherapy group $(97.8 \%, 73.9 \%)$. Events in $\geq 10 \%$ of the treated patients in either group are listed in Table $2 \mathrm{~b}$. 
Fig. 1 Kaplan-Meier plots of a overall survival, b PFS, and c DOR in the Japanese subpopulation. $C I$ confidence interval, $D O R$ duration of response, $H R$ hazard ratio, $P F S$ progressionfree survival

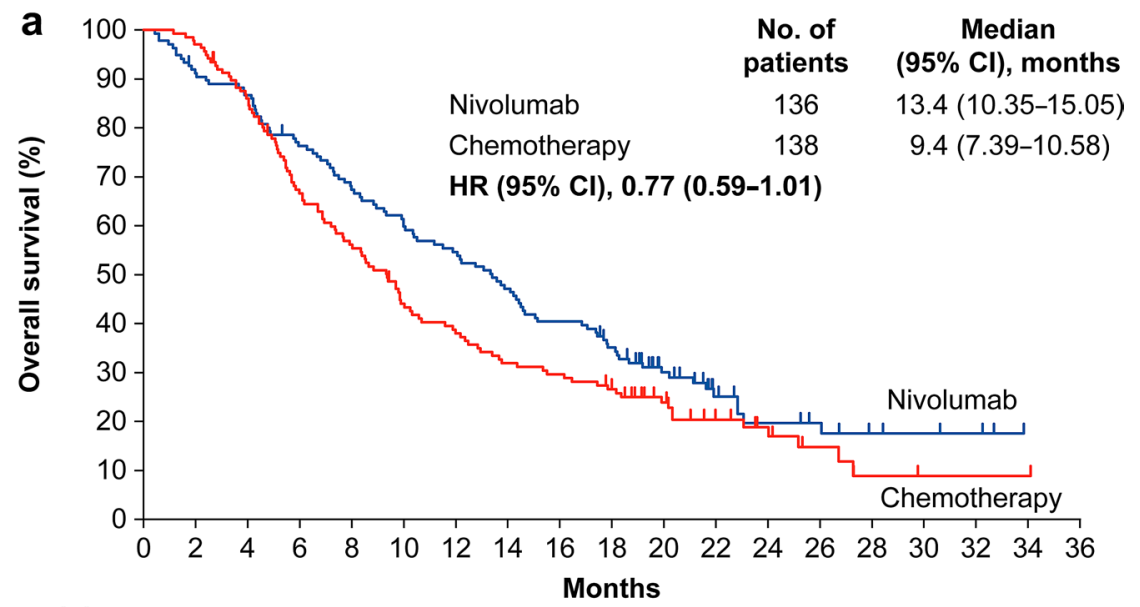

No. at risk

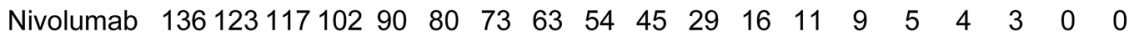

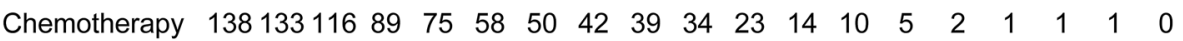
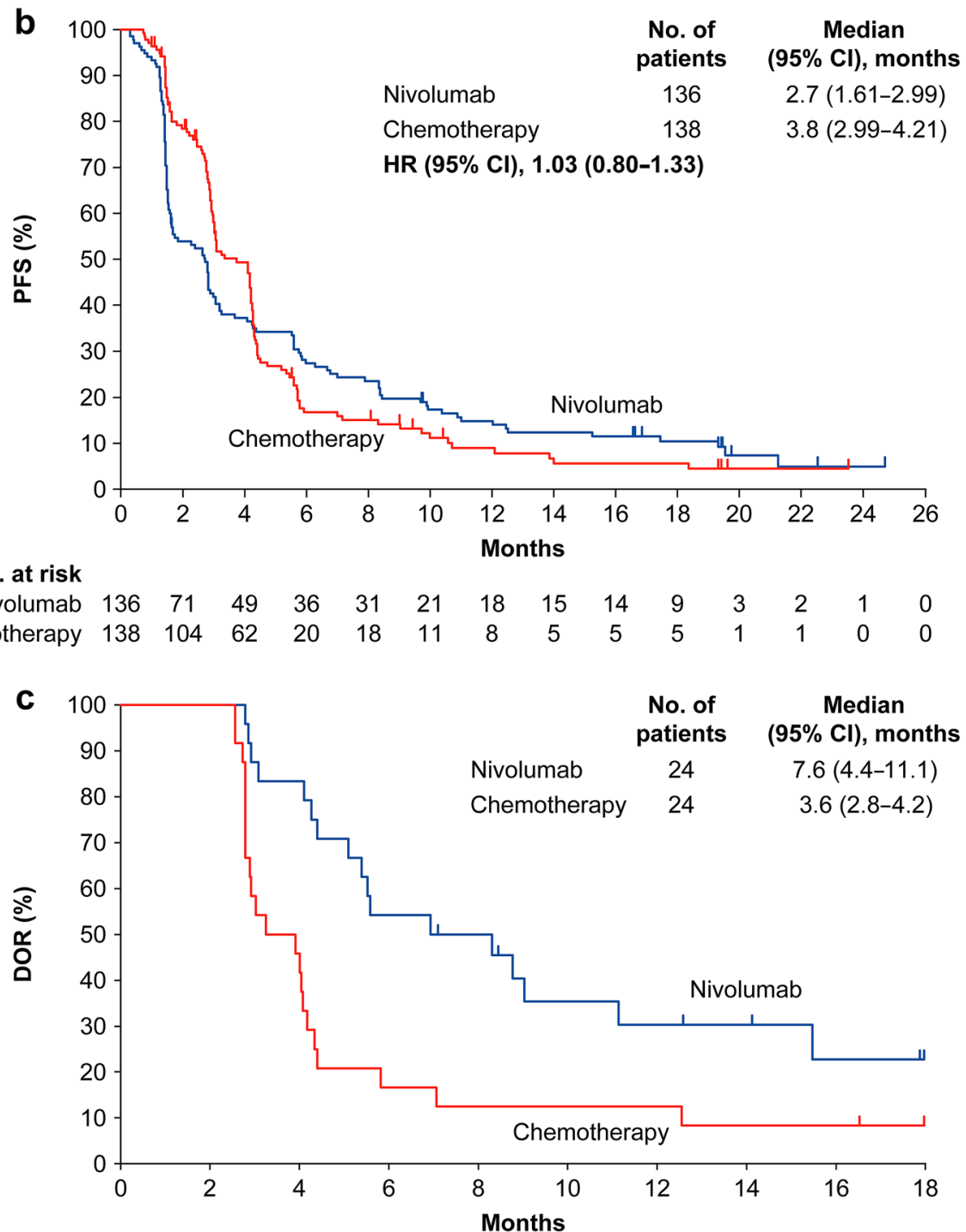

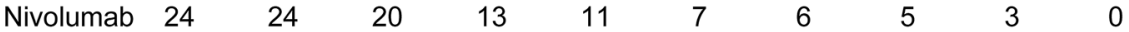

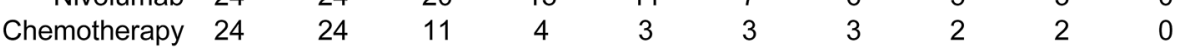



survival according to patient subgroups (Japanese subpopulation). ${ }^{\text {a}}$ Hazard ratios and their corresponding 95\% CIs for nivolumab relative to chemotherapy were calculated using the unstratified Cox proportional hazards model. CI confidence interval, ECOG Eastern Cooperative Oncology Group, $P D-L 1$ programmed death-ligand 1
Fig. 2 Forest plot of overall

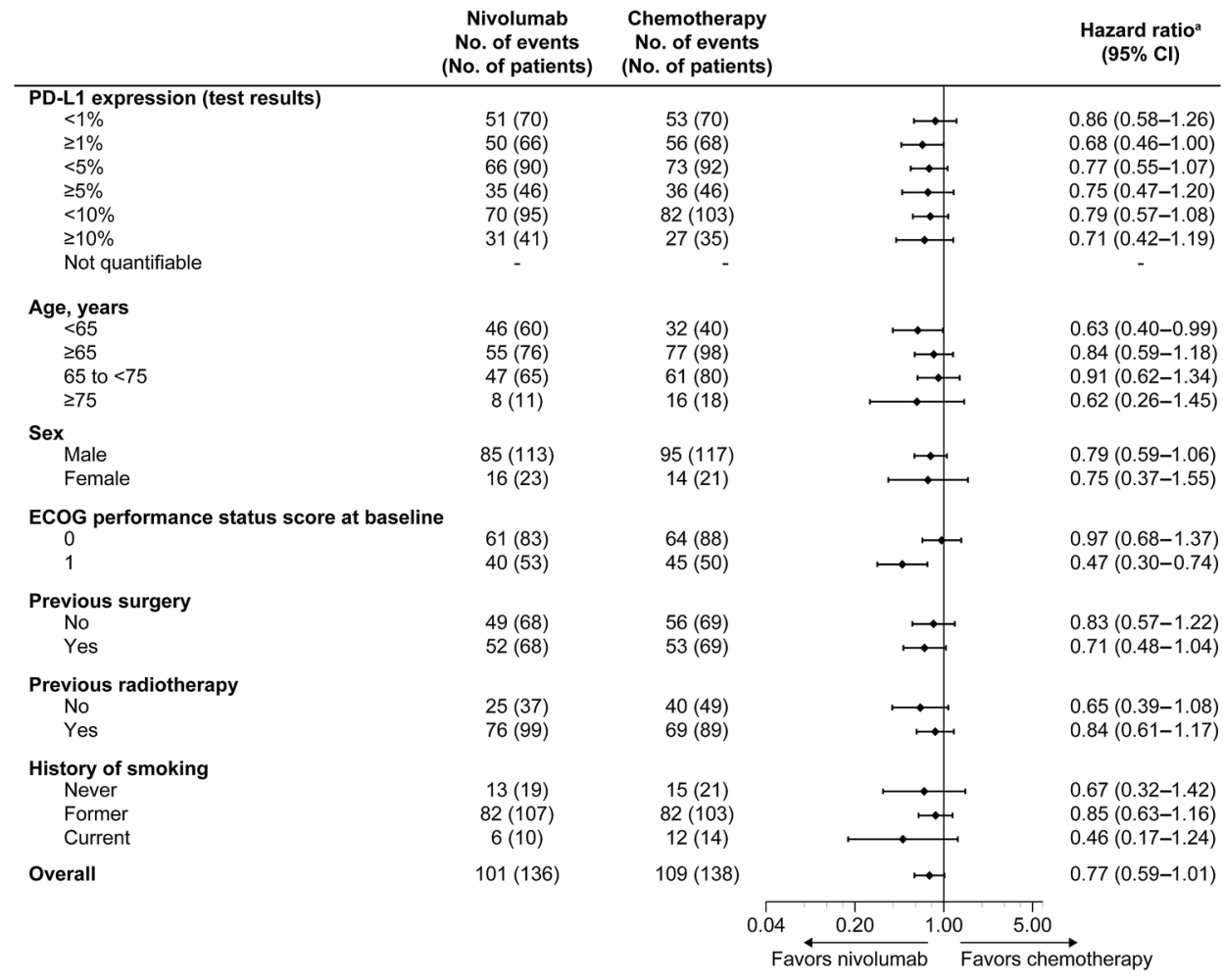

\section{Discussion}

To date, ICIs have been evaluated in patients with advanced esophageal cancer in two global studies (ATT RACTION-3 [14] and KEYNOTE-181 [16]). Of these studies, the proportion of Japanese patients was higher in ATTRACTION-3 [65.4\% (274/419)] than in KEYNOTE-181 [24.2\% (152/628)]. This sub-analysis of ATT RACTION-3 [14] is the first report demonstrating the efficacy and safety of nivolumab in a phase 3 trial in patients with esophageal cancer in Japan.

The baseline patient characteristics were mostly similar between the Japanese subpopulation and the overall ITT population [14]. However, more patients in the Japanese subpopulation had an ECOG PS of 0 in both groups versus the overall ITT population [61.0\% (83/136) vs. $48.1 \%$ (101/210)]. Median OS for nivolumab was numerically longer in the Japanese subpopulation (13.4 months) versus the overall ITT population (10.9 months); a similar trend in OS was also observed in the chemotherapy group (9.4 months vs. 8.4 months). In the subgroup analysis, OS was consistently numerically longer in the nivolumab group versus the chemotherapy group in the Japanese subpopulation, which was similar to the trend observed in the overall ITT population [14]. Further, the median PFS, ORR, and DCR in the Japanese subpopulation were numerically similar to those in the overall ITT population [14].
This trend in the improvement in OS may be attributed to a higher proportion of patients reporting activities of daily living and physical ability (ECOG PS, 0) in the Japanese subpopulation versus the overall ITT population. This trend in OS was also observed in nivolumab trials conducted in advanced melanoma and non-small cell lung cancer $[17,18]$. Patients with a good PS/clinical condition are also likely to receive subsequent treatment.

To this end, we observed differences in subsequent anticancer therapy that may have affected the results. After study treatment discontinuation, the proportion of patients who received subsequent anticancer treatment in the Japanese subpopulation and the overall ITT population was numerically higher in the nivolumab group [58.8\% (80/136) and $53 \%(112 / 210)]$ than in the chemotherapy group [47.1\% (65/138) and 47\% (99/209)]. Furthermore, the proportion of patients receiving taxanes as subsequent anticancer therapy in the nivolumab group was higher in the Japanese subpopulation versus the overall ITT population [56.6\% (77/136) vs. $48 \%$ (100/210)]; 66/75 patients who received PTX as subsequent anticancer therapy were Japanese. The reason for high PTX use in the Japanese population can be explained by the approval status and guideline description of PTX in Japan. PTX has been approved for the treatment of esophageal cancer and described in the guidelines [7] based on the results of a phase 2 study conducted in Japan [8]. Regarding the patients enrolled from other countries in ATTRAC TION-3 [14], PTX is not approved in Korea or Taiwan [19], 
Table 2 Response and disease control (a) and summary of treatment-related adverse events (b) in the Japanese subpopulation

\begin{tabular}{|c|c|c|c|c|c|c|c|c|}
\hline \multirow[t]{2}{*}{$n(\%)$} & \multicolumn{8}{|c|}{ Japanese subpopulation (RES) } \\
\hline & \multicolumn{3}{|c|}{ Nivolumab $(n=107)$} & \multicolumn{3}{|c|}{ Chemotherapy $(n=108)$} & \multicolumn{2}{|c|}{ OR $(95 \% \mathrm{CI})$} \\
\hline \multicolumn{9}{|l|}{ (a) Response and disease control } \\
\hline ORR & \multicolumn{2}{|c|}{$24(22.4)$} & & \multicolumn{2}{|c|}{$24(22.2)$} & & \multicolumn{2}{|c|}{$0.98(0.52-1.87)$} \\
\hline $\mathrm{CR}$ & \multicolumn{2}{|c|}{0} & & \multicolumn{2}{|c|}{$2(1.9)$} & & & \\
\hline PR & \multicolumn{2}{|c|}{$24(22.4)$} & & \multicolumn{2}{|c|}{$22(20.4)$} & & & \\
\hline SD & \multicolumn{2}{|c|}{$20(18.7)$} & & \multicolumn{2}{|c|}{$48(44.4)$} & & & \\
\hline PD & \multicolumn{2}{|c|}{$58(54.2)$} & & \multicolumn{2}{|c|}{$32(29.6)$} & & & \\
\hline $\mathrm{NE}$ & \multicolumn{2}{|c|}{$5(4.7)$} & & \multicolumn{2}{|c|}{$4(3.7)$} & & & \\
\hline $\mathrm{DCR}(\mathrm{CR}+\mathrm{PR}+\mathrm{SD})$ & \multicolumn{2}{|c|}{$44(41.1)$} & & \multicolumn{2}{|c|}{$72(66.7)$} & & \multicolumn{2}{|c|}{$0.30(0.17-0.55)$} \\
\hline \multirow[t]{2}{*}{$n(\%)$} & \multicolumn{4}{|c|}{ Nivolumab $(n=135)$} & \multicolumn{4}{|c|}{ Chemotherapy $(n=138)$} \\
\hline & Grade $1 / 2$ & Grade 3 & Grade 4 & Grade 5 & Grade $1 / 2$ & Grade 3 & Grade 4 & Grade 5 \\
\hline (b) Summary of treatment-relat & Iverse even & & & & & & & \\
\hline All events & $69(51)$ & $20(15)$ & $3(2)$ & 0 & $33(24)$ & $66(48)$ & $34(25)$ & $2(1)$ \\
\hline Serious events & $9(7)$ & $12(9)$ & $2(1)$ & 0 & $5(4)$ & $21(15)$ & $5(4)$ & $2(1)$ \\
\hline Events leading to discontinuation & $9(7)$ & $7(5)$ & 0 & 0 & $5(4)$ & $7(5)$ & $2(1)$ & $1(<1)$ \\
\hline Events leading to death & 0 & $2(1)$ & 0 & 0 & 0 & $1(<1)$ & 0 & $2(1)$ \\
\hline Events in $\geq 10 \%$ of treated patient & ither group & & & & & & & \\
\hline Rash & $15(11)$ & 0 & 0 & 0 & $18(13)$ & 0 & 0 & 0 \\
\hline Hypothyroidism & $14(10)$ & 0 & 0 & 0 & 0 & 0 & 0 & 0 \\
\hline Pyrexia & $10(7)$ & $1(<1)$ & 0 & 0 & $15(11)$ & 0 & 0 & 0 \\
\hline Diarrhoea & $8(6)$ & $1(<1)$ & 0 & 0 & $12(9)$ & $2(1)$ & 0 & 0 \\
\hline Fatigue & $8(6)$ & 0 & 0 & 0 & $21(15)$ & $4(3)$ & 0 & 0 \\
\hline Malaise & $8(6)$ & 0 & 0 & 0 & $42(30)$ & 0 & 0 & 0 \\
\hline Decreased appetite & $7(5)$ & 0 & 0 & 0 & $32(23)$ & $7(5)$ & 0 & 0 \\
\hline Stomatitis & $4(3)$ & 0 & 0 & 0 & $20(14)$ & $1(<1)$ & 0 & 0 \\
\hline Dysgeusia & $3(2)$ & 0 & 0 & 0 & $14(10)$ & 0 & 0 & 0 \\
\hline Lymphocyte count decreased & $2(1)$ & $2(1)$ & 0 & 0 & $6(4)$ & $9(7)$ & $3(2)$ & 0 \\
\hline Alopecia & $2(1)$ & 0 & 0 & 0 & $82(59)$ & 0 & 0 & 0 \\
\hline Arthralgia & $2(1)$ & 0 & 0 & 0 & $16(12)$ & 0 & 0 & 0 \\
\hline Constipation & $2(1)$ & 0 & 0 & 0 & $14(10)$ & 0 & 0 & 0 \\
\hline Neutrophil count decreased & $2(1)$ & 0 & 0 & 0 & $13(9)$ & $27(20)$ & $26(19)$ & 0 \\
\hline White blood cell count decreased & $1(<1)$ & $1(<1)$ & 0 & 0 & $18(13)$ & $29(21)$ & $13(9)$ & 0 \\
\hline Nausea & $1(<1)$ & 0 & 0 & 0 & $22(16)$ & 0 & 0 & 0 \\
\hline Neutropenia & $1(<1)$ & 0 & 0 & 0 & $6(4)$ & $16(12)$ & $6(4)$ & 0 \\
\hline Peripheral sensory neuropathy & $1(<1)$ & 0 & 0 & 0 & $42(30)$ & $1(<1)$ & 0 & 0 \\
\hline Anaemia & 0 & $3(2)$ & 0 & 0 & $20(14)$ & $16(12)$ & 0 & 0 \\
\hline Febrile neutropenia & 0 & 0 & 0 & 0 & 0 & $14(10)$ & $1(<1)$ & 0 \\
\hline Neuropathy peripheral & 0 & 0 & 0 & 0 & $15(11)$ & 0 & 0 & 0 \\
\hline
\end{tabular}

The deaths were caused by interstitial lung disease and pneumonitis in the nivolumab group and by pneumonia, spinal cord abscess, and interstitial lung disease in the chemotherapy group. Some patients had adverse events lower than grade 5 that subsequently led to death

$C I$ confidence interval, $C R$ complete response, $D C R$ disease control rate, $N E$ not evaluable, $O R$ odds ratio, $O R R$ objective response rate, $P D$ progressive disease, $P R$ partial response, $R E S$ response-evaluable set, $S D$ stable disease

and the proportion of patients from the remaining countries was limited. Therefore, PTX is considered to be more frequently used in the Japanese population than in the ITT population in ATTRACTION-3 and might have influenced OS. ICI treatment followed by chemotherapy reportedly has a favorable outcome [20,21], suggesting that the improved OS outcomes for nivolumab in the Japanese subpopulation could be attributed to the differences in subsequent anticancer therapies used as third-line treatment. Considering that OS may be indicative of the efficacy of both nivolumab and 
subsequent therapy, it is hypothesized that OS, and not PFS, is likely to be different between the Japanese population and overall ITT population based on the higher proportion of patients who received taxanes as subsequent therapy in the Japanese subpopulation than in the overall ITT population. Our data, however, showed that the HR for OS was the same in the Japanese subpopulation (0.77) and the overall ITT population (0.77), while the HR for PFS was similar in the Japanese subpopulation (1.03) and the overall ITT population (1.08). Based on these results, the difference in subsequent treatment regimens seems to have little impact on OS in the Japanese population and the overall ITT population. Alternatively, the difference in the proportion between the Japanese population and the overall ITT population is not enough to detect the differential effects of subsequent regimens. Therefore, it may be desirable to evaluate the effect of ICIs on subsequent chemotherapy regimens in the near future.

No notable difference in the efficacy and safety of nivolumab was observed between the Japanese subpopulation and the overall ITT population enrolled in ATTRAC TION-3 [14]. Similar subgroup analyses in Japanese or Asian subpopulations who received nivolumab for other cancers were consistent with those reported for the overall ITT population [22-24].

\section{Conclusion}

In the Japanese subpopulation, the OS was numerically longer for nivolumab versus chemotherapy, which was similar to the trend observed in the overall ITT population. The frequency of PTX use in the post-study treatment was different between the Japanese subpopulation and the overall ITT population, but this difference in the treatment environment was not clearly reflected in the study results. Additionally, no notable difference was observed between the safety profiles of the Japanese subpopulation and those of the overall ITT population. Nivolumab represents a new standard second-line treatment option for Japanese patients with advanced ESCC refractory to prior fluoropyrimidinebased chemotherapy.

\footnotetext{
Acknowledgements We thank the patients, their families, and the investigators. From Ono Pharmaceutical Co., Ltd., we thank medical monitors Yoshinobu Namba and Yoshinori Hirashima; clinical development team members Mitsunobu Tanimoto and Yasuhiro Matsumura; and Akira Takazawa for providing statistical support. Editorial support, in the form of medical writing, assembling tables and creating high-resolution images based on authors' detailed directions, collating author comments, copyediting, fact-checking, and referencing, was provided by Annirudha Chillar, MD, PhD, of Cactus Life Sciences (part of Cactus Communications) and funded by Ono Pharmaceutical Co., Ltd., Osaka, Japan, and Bristol-Myers Squibb, Princeton, NJ, USA. Statistical analysis support was provided by EPS Corporation.
}

\section{Compliance with ethical standards}

Ethical statement All procedures followed were in accordance with the ethical standards of the responsible committee on human experimentation (institutional and national) and with the Helsinki Declaration of 1964 and later versions. Informed consent or substitute for it was obtained from all patients for being included in the study.

Conflict of interest Masanobu Takahashi received grants from Ono Pharmaceutical Co., Ltd., during the conduct of the study; and personal fees from Ono Pharmaceutical Co., Ltd., Bristol-Myers Squibb K.K., Daiichi Sankyo, and Taiho Pharmaceutical Co., Ltd., outside the submitted work. Ken Kato received grants from Ono Pharmaceutical Co., Ltd., and Bristol-Myers Squibb K.K. during the conduct of the study; and research funds from MSD K.K., Shionogi \& Co. Ltd., BeiGene, Merck Biopharma Co., Ltd, and Oncolys BioPharma, outside the submitted work. Morihito Okada received grants and personal fees from Ono Pharmaceutical Co., Ltd., and Bristol-Myers Squibb K.K. during the conduct of the study. Yuichiro Doki received grants and personal fees from Ono Pharmaceutical Co., Ltd.; and grants from Bristol-Myers Squibb K.K. during the conduct of the study. Shigenori Kadowaki received grants and personal fees from Ono Pharmaceutical Co., Ltd., and Bristol-Myers Squibb K.K. during the conduct of the study; and grants and personal fees from Eli Lilly Japan K.K. and Chugai Pharmaceutical Co., Ltd.; personal fees from Bayer Yakuhin, Ltd., Daiichi Sankyo, Yakult Honsha Co., Ltd., Eisai Co., Ltd, and Merck KGaA; and grants from MSD K.K., Nobelpharma Co., Ltd, and Taiho Pharmaceutical Co., Ltd., outside the submitted work. Yasuo Hamamoto, Yutaro Kubota, Keisho Chin, Takashi Ogata, Yuichiro Nakashima, Ryu Ishihara, Masahiro Tsuda, and Satoru Motoyama received grants from Ono Pharmaceutical Co., Ltd., and Bristol-Myers Squibb K.K. during the conduct of the study. Hisato Kawakami received grants from Ono Pharmaceutical Co., Ltd. and Bristol-Myers Squibb K.K. during the conduct of the study; grants, personal fees, and other [advisory role, honoraria (speeches)] from Taiho Pharmaceutical Co., Ltd.; grants, personal fees, and other [advisory role, honoraria (speeches), research funding to institution] from Daiichi Sankyo; grants and personal fees (honoraria [speeches], research funding to institution) from Chugai Pharmaceutical Co., Ltd./Roche; personal fees and other [advisory role, honoraria (speeches)] from Bristol-Myers Squibb K.K., Ono Pharmaceutical Co., Ltd., Eli Lilly Japan K.K.; personal fees (honoraria [speeches]) from Yakult Honsha Co., Ltd., Takeda Pharmaceutical Company Limited, MSD K.K., Merck Serono Co., Ltd, Bayer Yakuhin, Ltd., and AstraZeneca K.K.; and grants (research funding to institution) from Eisai Co., Ltd, outside the submitted work. Hiroki Hara received grants from Ono Pharmaceutical Co., Ltd., and Bristol-Myers Squibb K.K. during the conduct of the study, and grants from AstraZeneca K.K., Sumitomo Dainippon Pharma Co., Ltd., Merck Biopharma Co., Ltd, Eisai Co., Ltd, LSK BioPharma, Incyte Corporation, Pfizer, Nippon Boehringer Ingelheim Co., Ltd., BeiGene, and Astellas; grants and personal fees from Daiichi Sankyo, MSD K.K., Taiho Pharmaceutical Co., Ltd., Chugai Pharmaceutical Co., Ltd., Ono Pharmaceutical Co., Ltd., and Bristol-Myers Squibb K.K.; and personal fees from Eli Lilly Japan K.K., Yakult Honsha Co., Ltd., Sanofi K.K., Takeda Pharmaceutical Company Limited, Kyowa Kirin Co., Ltd., outside the submitted work. Manabu Muto received grants from Ono Pharmaceutical Co., Ltd., and Bristol-Myers Squibb K.K. during the conduct of the study, and grants from Taiho Pharmaceutical Co., Ltd., Chugai Pharmaceutical Co., Ltd., Yakult Honsha Co., Ltd., Sysmex Corporation, Riken Genesis, Mitsui Knowledge Inc., and Ono Pharmaceutical Co., Ltd., outside the submitted work. Mamoru Kodani is an employee of Ono Pharmaceutical Co., Ltd. Yuko Kitagawa received grants and personal fees from Asahi Kasei Pharma Corp., Taiho Pharmaceutical Co., Ltd., Chugai Pharmaceutical Co., Ltd., EA Pharma Co., Ltd., Yakult Honsha Co., Ltd., Otsuka Pharmaceutical 
Co., Ltd., Otsuka Pharmaceutical Factory Inc., Shionogi \& Co., Ltd., Astellas Pharma Inc., Sumitomo Dainippon Pharma Co., Ltd., Taisho Toyama Pharmaceutical Co., Ltd., Ono Pharmaceutical Co., Ltd., Nihon Pharmaceutical Co., Ltd., and Sanofi K.K.; and grants from Daiichi Sankyo Company, Limited, Merck Serono Co., Ltd., Takeda Pharmaceutical Company Limited, Kaken Pharmaceutical Co., Ltd., Kowa Pharmaceutical Co., Ltd., Medicon Inc., Kyowa Kirin Co., Ltd., Pfizer Japan Inc., Japan Blood Products Organization, Medtronic Japan Co., Ltd., Eisai Co., Ltd., Tsumura and Co., KCI Licensing, Inc., Abbott Japan Co., Ltd., Fujifilm Toyama Chemical Co., Ltd., Nippon Covidien Inc., outside the submitted work.

Open Access This article is licensed under a Creative Commons Attribution 4.0 International License, which permits use, sharing, adaptation, distribution and reproduction in any medium or format, as long as you give appropriate credit to the original author(s) and the source, provide a link to the Creative Commons licence, and indicate if changes were made. The images or other third party material in this article are included in the article's Creative Commons licence, unless indicated otherwise in a credit line to the material. If material is not included in the article's Creative Commons licence and your intended use is not permitted by statutory regulation or exceeds the permitted use, you will need to obtain permission directly from the copyright holder. To view a copy of this licence, visit http://creativecommons.org/licenses/by/4.0/.

\section{References}

1. American Cancer Society. 2020. https://cancerstatisticscent er.cancer.org/\#!/. Accessed 20 Apr 2020.

2. Cancer Information Service. Cancer statistics in Japan-survival. https://ganjoho.jp/en/professional/statistics/table_download.html. Accessed 20 Apr 2020.

3. Abnet CC, Arnold M, Wei WQ. Epidemiology of esophageal squamous cell carcinoma. Gastroenterology. 2018;154:360-73.

4. Bray F, Ferlay J, Soerjomataram I, et al. Global cancer statistics 2018: GLOBOCAN estimates of incidence and mortality worldwide for 36 cancers in 185 countries. CA Cancer J Clin. 2018;68:394-424.

5. Zhang HZ, Jin GF, Shen HB. Epidemiologic differences in esophageal cancer between Asian and Western populations. Chin J Cancer. 2012;31:281-6.

6. Kitagawa Y, Uno T, Oyama T, et al. Esophageal cancer practice guidelines 2017 edited by the Japan Esophageal Society: part 1 . Esophagus. 2019a;16:1-24.

7. Kitagawa Y, Uno T, Oyama T, et al. Esophageal cancer practice guidelines 2017 edited by the Japan esophageal society: part 2 . Esophagus. 2019b;16:25-43.

8. Kato K, Tahara M, Hironaka S, et al. A phase II study of paclitaxel by weekly 1-h infusion for advanced or recurrent esophageal cancer in patients who had previously received platinum-based chemotherapy. Cancer Chemother Pharmacol. 2011;67:1265-72.

9. Muro K, Hamaguchi T, Ohtsu A, et al. A phase II study of singleagent docetaxel in patients with metastatic esophageal cancer. Ann Oncol. 2004;15:955-9.

10. Jimenez P, Pathak A, Phan AT. The role of taxanes in the management of gastroesophageal cancer. J Gastrointest Oncol. 2011;2:240-9.
11. American Cancer Society. Esophagus cancer. https://www.cance r.org/cancer/esophagus-cancer.html. Accessed 20 Apr 2020.

12. Kudo T, Hamamoto Y, Kato K, et al. Nivolumab treatment for oesophageal squamous-cell carcinoma: an open-label, multicentre, phase 2 trial. Lancet Oncol. 2017;18:631-9.

13. Kato K, Doki Y, Ura T, et al. Long-term efficacy and predictive correlates of response to nivolumab in Japanese patients with esophageal cancer. Cancer Sci. 2020;111:1676-84.

14. Kato K, Cho BC, Takahashi M, et al. Nivolumab versus chemotherapy in patients with advanced oesophageal squamous cell carcinoma refractory or intolerant to previous chemotherapy (ATT RACTION-3): a multicentre, randomised, open-label, phase 3 trial. Lancet Oncol. 2019;20:1506-17.

15. Approval. https://www.fdanews.com/articles/196077-japan-appro ves-opdivo-for-unresectable-advanced-or-recurrent-esophageal -cancer Accessed 14 August 2020.

16. Metges J, Francois E, Shah M, et al. The phase 3 KEYNOTE-181 study: pembrolizumab versus chemotherapy as second-line therapy for advanced esophageal cancer. Ann Oncol. 2019;30(Suppl 4):iv130.

17. Nakamura Y, Kitano S, Takahashi A, et al. Nivolumab for advanced melanoma: pretreatment prognostic factors and early outcome markers during therapy. Oncotarget. 2016;7:77404-15.

18. Oya Y, Yoshida T, Kuroda H, et al. Predictive clinical parameters for the response of nivolumab in pretreated advanced non-smallcell lung cancer. Oncotarget. 2017;8:103117-28.

19. Muro K, Lordick F, Tsushima T, et al. Pan-Asian adapted ESMO Clinical Practice Guidelines for the management of patients with metastatic oesophageal cancer: a JSMO-ESMO initiative endorsed by CSCO, KSMO, MOS, SSO and TOS Ann Oncol. 2019;30(1):34-43.

20. Saleh K, Daste A, Martin N, et al. Response to salvage chemotherapy after progression on immune checkpoint inhibitors in patients with recurrent and/or metastatic squamous cell carcinoma of the head and neck. Eur J Cancer. 2019;121:123-9.

21. Togasaki K, Sukawa Y, Kanai T, et al. Clinical efficacy of immune checkpoint inhibitors in the treatment of unresectable advanced or recurrent gastric cancer: an evidence-based review of therapies. Onco Targets Ther. 2018;11:8239-50.

22. Kato K, Satoh T, Muro K, et al. A subanalysis of Japanese patients in a randomized, double-blind, placebo-controlled, phase 3 trial of nivolumab for patients with advanced gastric or gastro-esophageal junction cancer refractory to, or intolerant of, at least two previous chemotherapy regimens (ONO-4538-12, ATTRACTION-2). Gastric Cancer. 2019;22:344-54.

23. Tomita Y, Fukasawa S, Shinohara N, et al. Nivolumab versus everolimus in advanced renal cell carcinoma: Japanese subgroup 3-year follow-up analysis from the phase III CheckMate 025 study. Jpn J Clin Oncol. 2019;49:506-14.

24. Kiyota N, Hasegawa Y, Takahashi S, et al. A randomized, openlabel, phase III clinical trial of nivolumab vs. therapy of investigator's choice in recurrent squamous cell carcinoma of the head and neck: a subanalysis of Asian patients versus the global population in checkmate 141. Oral Oncol. 2017;73:138-46.

Publisher's Note Springer Nature remains neutral with regard to jurisdictional claims in published maps and institutional affiliations. 


\section{Affiliations}

Masanobu Takahashi ${ }^{1} \cdot \mathrm{Ken} \mathrm{Kato}^{2} \cdot$ Morihito Okada $^{3} \cdot \mathrm{Keisho} \mathrm{Chin}^{4} \cdot$ Shigenori Kadowaki $^{5} \cdot$ Yasuo Hamamoto $^{6}$. Yuichiro Doki ${ }^{7}$. Yutaro Kubota ${ }^{8} \cdot$ Hisato Kawakami $^{9} \cdot$ Takashi Ogata $^{10}$ - Hiroki Hara ${ }^{11}$. Manabu Muto ${ }^{12}$. Yuichiro Nakashima $^{13} \cdot$ Ryu Ishihara ${ }^{14} \cdot$ Masahiro Tsuda $^{15} \cdot$ Satoru Motoyama ${ }^{16} \cdot$ Mamoru Kodani $^{17} \cdot$ Yuko Kitagawa $^{18}$

1 Department of Medical Oncology, Tohoku University Hospital, 1-1 Seiryo-machi, Aoba-ku, Sendai-shi, Miyagi 980-8574, Japan

2 Department of Gastrointestinal Medical Oncology, National Cancer Center Hospital, Tokyo, Japan

3 Department of Surgical Oncology, Research Institute for Radiation Biology and Medicine, Hiroshima University, Hiroshima, Japan

4 Department of Gastroenterological Chemotherapy, The Cancer Institute Hospital of Japanese Foundation for Cancer Research, Tokyo, Japan

5 Department of Clinical Oncology, Aichi Cancer Center Hospital, Nagoya, Japan

6 Keio Cancer Center, Keio University School of Medicine, Tokyo, Japan

7 Department of Surgery, Osaka University Hospital, Osaka, Japan

8 Department of Medicine, Division of Medical Oncology, Showa University School of Medicine, Tokyo, Japan

9 Department of Medical Oncology, Kindai University Faculty of Medicine, Osakasayama, Osaka, Japan
10 Department of Gastrointestinal Surgery, Kanagawa Cancer Center, Yokohama, Japan

11 Department of Gastroenterology, Saitama Cancer Center, Saitama, Japan

12 Department of Clinical Oncology, Kyoto University Hospital, Kyoto, Japan

13 Department of Surgery and Science, Graduate School of Medical Sciences, Kyushu University, Fukuoka, Japan

14 Department of Gastrointestinal Oncology, Osaka International Cancer Institute, Osaka, Japan

15 Department of Gastroenterological Oncology, Hyogo Cancer Center, Akashi, Japan

16 Department of Thoracic Surgery, Akita University Graduate School of Medicine, Akita, Japan

17 Department of Oncology, Ono Pharmaceutical Co., Ltd., Osaka, Japan

18 Department of Surgery, Keio University School of Medicine, Tokyo, Japan 